\title{
THE PRINCIPAL FIBRE BUNDLE ON LORENTZIAN ALMOST R-PARA CONTACT STRUCTURE
}

\author{
Lovejoy S. Das ${ }^{1, a}$ and Mohammad Nazrul Islam Khan ${ }^{2, *}$
}

\footnotetext{
${ }^{1}$ Department of Mathematics, Kent State University, New Philadelphia, OH 44663, USA

a ldas@kent.edu

${ }^{2}$ Qassim University, Buraidah-51452, P.O. Box 6688, Saudi Arabia

Communicated by László Tóth
}

\section{Original Research Paper}

Received: Mar 30, 2020 • Accepted: Nov 27, 2020

First published online: Mar 24, 2021

๑) 2020 The Author(s)

\section{ABSTRACT}

The purpose of this paper is to study the principal fibre bundle $\left(P, M, G, \pi_{p}\right)$ with Lie group $G$, where $M$ admits Lorentzian almost paracontact structure $\left(\phi, \xi_{p}, \eta_{p}, g\right)$ satisfying certain condtions on $(1,1)$ tensor field $J$, indeed possesses an almost product structure on the principal fibre bundle. In the later sections, we have defined trilinear frame bundle and have proved that the trilinear frame bundle is the principal bundle and have proved in Theorem 5.1 that the Jacobian map $\pi^{*}$ is the isomorphism.

\section{KEYWORDS}

principal fibre bundle, Lie group, trilinear frame, Cartesian product manifold, linear connections, almost product structure, projection map

\section{MATHEMATICS SUBJECT CLASSIFICATION (2020)}

Primary 53D15; Secondary 57R25

\section{INTRODUCTION}

The theory of principal fibre bundles was developed by S. Kobayashi [7], S. Kobayashi and K. Nomizu [6], Bishop and Critterdon [1] and many others. Principal fibre bundles have played an important role in differential geometry and mathematical physics. Motivated by the works of Boothby-Wong [2] and K. Taleb [9], the authors have defined and studied Lorentzian almost r-paracontact structure [5]. In Section 2, we recall basic definitions of principal fibre bundles, Lie algebra and general linear group. Section 3 deals with Lorentzian almost r-para-contact structure and almost product structure. In Section 4, we have proved that the trilinear frame bundle is the principal fibre bundle while the last Section is dedicated to proving that the horizontal subspace is isomorphic to the tangent space of the product manifold by the Jacobian map of the projection map.

\footnotetext{
*Corresponding author. E-mail: nazrul@qu.edu.sa,mnazrul@rediffmail.com
} 


\section{PRELIMINARIES}

A set $(P, M, G, \pi)$ is called the principal fibre bundle if $P$ is a differentiable manifold, $G$ a Lie group and

(i) $G$ acts on $P$ differentiably to the right i.e. there exists a differentiable map $P \times G \rightarrow P$ such that $(u, g) \rightarrow u g$ where $u \in P, g \in G$ and $u g \in P$ where $(u g) h=u(g h)$ for all $g, h \in G$.

(ii) $M$ is the quotient manifold $P=G$ and the projection map: $P / M$ is differentiable.

(iii) For each $x \in M$ and for every neighborhood $U$ of $x$, the set $\pi^{-1}(U)$ is isomorphic to $U \times G$ [4].

Let $G$ be a $C^{\infty}$ manifold. If $G$ is a group and the maps

(i) $G \times G \rightarrow G$ such that $\left(g_{1}, g_{2}\right) \rightarrow g_{1} g_{2}$ and

(ii) $G \rightarrow G$ such that $g, g^{-1}$ are differentiable, then $G$ is called the Lie group. Here $g_{1}, g_{2}$ and $g$ are arbitrary elements of $G$.

If $G l(n, R)$ be the set of all $n \times n$ non-singular matrices over $R$, then $G l(n, R)$ is a group under matrix multiplication. If $g \in G l(n, R), g$ can be expressed as $\left(g_{b}^{a}\right), g_{b}^{a} \in R . g_{b}^{a}$ can be treated as coordinates and induce the manifold structure in $G l(n, R)$ and $G l(n, R)$ is a Lie group. It is called general linear group.

Let $G$ be a Lie group and let $g \rightarrow G$. Then the map $L_{g}: G \rightarrow G$ such that $L_{g}(h)=g h, h \in G$ is an automorphism of $G$ called left translation. If $X$ is a vector field on $G$ such that $(L g)_{*} X=X, X$ is called left invariant vector field where $(L g)_{*}$ denotes Jacobian map of $L g$.

Let $G$ be a Lie group and $S$ be the set of vector fields over $G$. If for all $X, Y \in S,[X ; Y] \in S$ such that

$$
\begin{aligned}
& \text { (i) }[X, Y]=-[Y, X] \\
& \text { (ii) } \quad[[X, Y], Z]+[[Y, Z], X]+[[Z, X], Y]=0
\end{aligned}
$$

for all $X, Y, Z \in S$, then $S$ is called Lie algebra over $G$. It is easy to show that the set of all left invariant vector fields form the Lie Algebra over $G$ [10].

\section{LORENTZIAN ALMOST R-PARA-CONTACT STRUCTURE}

Let $M$ be a differentiable manifold of $C^{\infty}$ class and $T(M)$ denotes the tangent bundle of $M$. Suppose that there are given a tensor field $\phi$ of type $(1,1)$, a vector field $\xi_{p}$ and a 1 -form $\eta_{p}, p=1,2, \ldots, r$ satisfying [3]

$$
\begin{aligned}
& \phi^{2}=I-\sum_{p=1}^{r} \xi_{p} \otimes \eta_{p} \\
& \phi \xi_{p}=0 \\
& \text { (iii) } \quad \eta_{p} \circ \phi=0
\end{aligned}
$$$$
\text { (iv) } \quad \eta_{p}\left(\xi_{q}\right)=\delta_{p q}
$$

where $p=1,2, \ldots, r$ and $\delta_{p q}$ denote the Kronecker delta. Thus the manifold $M$ satisfying conditions (3.1) will be said to possess Lorentzian almost r-para-contact structure $[5,8]$.

THEOREM 3.1. Let $\left(P, M, G, \pi_{p}\right)$ be the principal fibre bundle with Lie group $G$. The $(1,1)$ tensor field $J$ satisfying

$$
\text { (i) } \quad \pi(J X)=\phi \pi X-\left\{a \overline{\eta_{p}}(\omega X)+b \eta_{p}(\pi X)\right\} \xi_{p}
$$

and

$$
\text { (ii) } \quad \omega(J X)=\phi \omega X-\left\{a^{-1}\left(1-b^{2}\right) \eta_{p}(\pi X)-b \overline{\eta_{p}}(\omega X)\right\} \xi_{p}
$$

gives an almost product structure on $P$.

Proof. Let $\left(P, M, G, \pi_{p}\right)$ be the principal fibre bundle with Lie group $G$ and the projection map $\pi_{p}$ and let $\omega$ be the connection 1-form in $P$. Let $u^{h}$ and $A *$ be lift of $u \in \chi(M)$ and the fundamental vector field with respect to $A \in \wp$ where $\wp$ is the Lie algebra of the Lie group $G$. Suppose further that $M$ admits Lorentzian almost $r$-paracontact structure $\left(\phi, \xi_{p}, \eta_{p}, g\right)$. Let $\left(\bar{\phi}, \overline{\xi_{p}}, \bar{\eta}_{p}\right)$ be the left invariant 
Lorentzian almost $r$-paracontact structure over the Lie group $G$. Let $\omega$ be connection 1-form on $P$. For a tensor field $J$ of type $(1,1)$ on $P$, we define the structure on $M$ as

(i) $\quad \pi(J X)=\phi \pi X-\left\{a \bar{\eta}_{p}(\omega X)+b \eta_{p}(\pi X)\right\} \xi_{p}$

and

$$
\text { (ii) } \quad \omega(J X)=\phi \omega X-\left\{a^{-1}\left(1-b^{2}\right) \eta_{p}(\pi X)-b \overline{\eta_{p}}(\omega X)\right\} \xi_{p}
$$

where $a$ and $b$ are real numbers. Then we can easily verify that

$$
\pi\left(J^{2} X\right)=\pi X
$$

and

$$
\omega\left(J^{2} X\right)=\omega X
$$

hence $J$ gives an almost product structure on $P$.

THEOREM 3.2. Let $\left(P, M, G, \pi_{p}\right)$ be the principal fibre bundle with Lie group $G$. The $(1,1)$ tensor field $J$ satisfying

$$
\begin{aligned}
J X^{*} & =(\bar{\phi} A)^{*}-a \eta_{p}(A) \xi_{p}^{h}-b \bar{\eta}_{p}(\bar{A}) \xi_{p}^{*} \\
J u^{*} & =(\phi u)^{h}+b \eta_{p}(u) \xi_{p}^{h}-a^{-1}\left(1-b^{2}\right) \eta_{p}(u) \xi_{p}^{*}
\end{aligned}
$$

gives an almost product structure on $P$.

Proof. For $A, B \in S, S$ the Lie algebra over the Lie group $G$ and for $u, v \in \chi(M)$, we have [3]

$$
\begin{aligned}
\text { (i) } & {\left[A^{*} u^{h}\right] } & =0 \\
\text { (ii) } & {\left[A^{*}, B^{*}\right] } & =[A, B]^{*} \\
\text { (iii) } & {\left[u^{h}, v^{h}\right] } & =-\Omega\left(u^{h}, v^{h}\right)
\end{aligned}
$$

where $A^{*}$ is the fundamental vector field with respect to $A, u^{h}$ the horizontal lift and $\Omega$ the curvature form of the connection.

For the fundamental vector field $A^{*}$ and the horizontal lift $u^{h}$, let us define

(i) $\quad J X^{*}=(\bar{\phi} A)^{*}-a \eta_{p}(A) \xi_{p}^{h}-b \overline{\eta_{p}}\left(\bar{A} \xi_{p}^{*}\right.$

(ii) $\quad J u^{*}=(\phi u)^{h}+b \eta_{p}(u) \xi_{p}^{h}-a^{-1}\left(1-b^{2}\right) \eta_{p}(u) \xi_{p}^{*}$

then we can easily to verify that

$$
\begin{aligned}
& \text { (i) } J^{2} A^{*}=A^{*} \\
& \text { (ii) } \quad J^{2} \xi_{p}^{h}=\xi_{p}^{h}
\end{aligned}
$$

Hence $J$ again gives an almost product structure on $P$.

\section{TRILINEAR FRAME BUNDLE}

Let $M_{1}, M_{2}, M_{3}$ be three $C^{\infty}$ manifolds each of dimension $n$. If $x \in M_{1}, y \in M_{2}$ and $z \in M_{3}$ then $(x, y, z)$ is the point of cartesian product manifold $M_{1} \times M_{2} \times M_{3}$. Let $\left(x^{1}, x^{2}, \ldots, x^{n}\right),\left(y^{1}, y^{2}, \ldots, y^{n}\right)$ and $\left(z^{1}, z^{2}, \ldots, z^{n}\right)$ be local coordinate systems about $x, y, z$ in the manifolds $M_{1}, M_{2}$ and $M_{3}$ respectively. Then $\left\{\left(x^{i}, y^{j}, z^{k}\right), 1 \leq i, j, k \leq n\right\}$ of $n^{3}$ triplets forms the local coordinate system about $(x, y, z)$ in the product manifold. The canonical basis vectors about $(x, y, z)$ in $M_{1} \times M_{2} \times M_{3}$ are $\left\{\left(\frac{\partial}{\partial x^{i}}\right),\left(\frac{\partial}{\partial y^{j}}\right),\left(\frac{\partial}{\partial z^{k}}\right), 1 \leq i, j, k \leq n\right\}$.

If $U_{a}, V_{b}$ and $W_{c}$ be tangent vectors to $M_{1}, M_{2}, M_{3}$ at points $x, y, z$ respectively, we can write

$$
U_{\alpha}=U_{a}^{i} \frac{\partial}{\partial x^{i}}, \quad V_{\alpha}=V_{b}^{j} \frac{\partial}{\partial y^{j}}, \quad W_{\alpha}=W_{a}^{k} \frac{\partial}{\partial z^{k}}
$$

It is easy to show that the set $\left\{U_{a}, V_{b}, W_{c}, 1 \leq a, b, c \leq n\right\}$ is the set of $n^{3}$ vectors forming the basis of tangent space of the product manifold $M_{1} \times M_{2} \times M_{3}$. 
Let us call the set $\left\{x^{i}, y^{j}, z^{k}, U_{a}^{i}, V_{b}^{j}, W_{c}^{k}\right\}$ as the trilinear frame at $(x, y, z)$ of $M_{1} \times M_{2} \times M_{3}$. We denote by $T L$ the set of the trilinear frames at different points of the product manifold $M_{1} \times M_{2} \times M_{3}$. It can be shown that $T L$ is also a differentiable manifold.

Let $u=\left(x^{i}, y^{j}, z^{k}, U_{a}^{i}, V_{b}^{j}, W_{c}^{k}\right) \in T L$ and $g, h, p \in G l(n, R)$ where $g=\left(g_{b}^{a}\right), h=\left(h_{c}^{b}\right), p=\left(p_{d}^{c}\right)$. We define action of $G l(n, R) \times G l(n, R) \times G l(n, R)$ on $T L$ as follows

$$
\left(\left(x^{i}, y^{j}, z^{k}, U_{a}^{i}, V_{b}^{j}, W_{c}^{k}\right),\left(\left(g_{b}^{a}\right),\left(h_{c}^{b}\right),\left(p_{d}^{c}\right)\right) \rightarrow\left(x^{i}, y^{j}, z^{k}, U_{a}^{i} g_{d}^{a}, V_{b}^{j} h_{c}^{b}, W_{c}^{k} p_{f}^{c}\right)\right.
$$

It is mapping

$$
T L \times G l(n, R) \times G l(n, R) \times G l(n, R) \rightarrow T L .
$$

Let us call the set

$$
\left\{T L, M_{1} \times M_{2} \times M_{3}, \pi, G l(n, R) \times G l(n, R) \times G l(n, R)\right\}
$$

the trilinear frame bundle over the product manifold where $\pi$ is projection map.

THEOREM 4.1. The trilinear frame bundle is the principal fibre bundle.

Proof. To show that $\left\{T L, M_{1} \times M_{2} \times M_{3}, \pi, G l(n, R) \times G l(n, R) \times G l(n, R)\right\}$ is the principal fibre bundle, we have to show

(i) $G l(n, R) \times G l(n, R) \times G l(n, R)$ acts on $T L$ differentiably to the right. It is obvious by the equation (2.1). We can also define

$$
\left(x^{i}, y^{j}, z^{k}, U_{a}^{i} g_{d}^{a}, V_{b}^{j} h_{c}^{b}, W_{c}^{k} p_{f}^{c}\right)\left(\left(q_{b}^{a}\right),\left(r_{c}^{b}\right), s_{d}^{c}\right)=\left(x^{i}, y^{j}, z^{k}, U_{a}^{i}, V_{b}^{j}, W_{c}^{k}\right)\left(g_{d}^{a} q_{c}^{d}, h_{f}^{b} r_{l}^{f}, p_{m}^{c} s_{n}^{m}\right) .
$$

Hence $G l(n, R) \times G l(n, R) \times G l(n, R)$ acts on $T L$ differentiably to the right.

(ii) $M_{1} \times M_{2} \times M_{3}$ can be treated as the quotient manifold $T L / G l(n, R) \times G l(n, R) \times G l(n, R)$ and the projection map $\pi: T L \rightarrow M_{1} \times M_{2} \times M_{3}$ is differentiable.

(iii) Let $(x, y, z) \in M_{1} \times M_{2} \times M_{3}$ and $U$ be coordinate neighbourhood of $(x, y, z)$ in the product manifold. $\left\{\left(x^{i}, y^{j}, z^{k}\right), 1 \leq i, j, k \leq n\right\}$ is the local coordinate system in $U$. As $\pi^{-1}(U) \subset T L$, we can take

$$
\pi^{-1}(U)=\left\{\left(x^{i}, y^{j}, z^{k}, g_{b}^{a}, h_{d}^{c}, p_{f}^{c}\right)\right\} .
$$

As $\left(x^{i}, y^{j}, z^{k}\right) \in U$ and $\left(g_{b}^{a}, h_{d}^{c}, p_{f}^{c}\right) \in G l(n, R) \times G l(n, R) \times G l(n, R)$ so

$$
\left(x^{i}, y^{j}, z^{k}, g_{b}^{a}, h_{d}^{c}, p_{f}^{c}\right) \in U \times G l(n, R) \times G l(n, R) \times G l(n, R) .
$$

We can take the identity map

$$
I: \pi^{-1}(U) \rightarrow U \times G l(n, R) \times G l(n, R) \times G l(n, R)
$$

such that $\left(x^{i}, y^{j}, z^{k}, g_{b}^{a}, h_{d}^{c}, p_{f}^{c}\right) \rightarrow\left(x^{i}, y^{j}, z^{k}, g_{b}^{a}, h_{d}^{c}, p_{f}^{c}\right)$. Since identity map is always the isomorphism so $\pi^{-1}(U)$ is isomorphic to $U \times G l(n, R) \times G l(n, R) \times G l(n, R)$. Thus the trilinear frame bundle becomes the principal fibre bundle.

\section{LINEAR CONNECTIONS}

Let $\left\{T L, M_{1} \times M_{2} \times M_{3}, \pi, G l(n, R) \times G l(n, R) \times G l(n, R)\right\}$ be the trilinear frame bundle and for $u \in$ $T L, T u$ be tangent space of $T L$ at $u$. Let $L_{u}^{v}$ be the subspace of $T u$ such that

$$
L_{u}^{v}=\left\{X \in T \mid \pi^{*}(X)=0\right\},
$$

where $\pi^{*}$ is the jacobian map of $\pi$.

A connection $\Gamma$ on $T L$ is an assignment of the subspace $\Gamma_{u}^{h}$ of $T u$ such that

$$
T u=L_{u}^{v}+\Gamma_{u}^{h}
$$

Further

$$
T_{u} g^{h}=\left(R_{g}\right) * \Gamma_{u}^{h}
$$

where $R_{\mathrm{g}}$ is the right translation. Let us call $L_{u}^{v}$ and $\Gamma_{u}^{h}$ as vertical and horizontal subspaces of $T u$. We can express arbitrary $X \in T u$ as

where $Y \in L_{u}^{v}$ and $Z \in \Gamma_{u}^{h}$.

$$
X=Y+Z
$$


If $Z=0, X$ is called vertical vector field and if $Y=0, X$ is horizontal vector field.

THEOREM 5.1. The horizontal subspace $\Gamma_{u}^{h}$ is isomorphic to the tangent space $T_{p}$ of the product manifold at $p=\pi(u)$ by the Jacobian map $\pi^{*}$ of the projection map.

Proof. Since $\pi$ is the map $T L \rightarrow M_{1} \times M_{2} \times M_{3}$ so $\pi_{*}$ is the linear transformation $T u \rightarrow T_{p}$. But $\pi^{*} T_{u}=\pi^{*} \Gamma_{u}^{h}$ as $\pi^{*} L_{u}^{v}=0$. Thus $\pi^{*} \Gamma_{u}^{h} \subset T_{p}$ or $\pi^{*} T u \subset T_{p}$.

Now for arbitrary $X \in T p$, we can construct a tangent vector $(X ; 0 ; 0 ; 0)$ in $U \times G l(n, R) \times G l(n, R) \times$ $G l(n, R), 0$ null matrix of $G l(n ; R)$ and $U$ the coordinate neighborhood of $p$ in $M_{1} \times M_{2} \times M_{3}$. Since trilinear frame bundle is the principal fibre bundle, $\pi^{-1}(U)$ is isomorphic to $U \times G l(n, R) \times G l(n, R)$ and isomorphism is the identity map. So we can assume that $(X, 0,0,0) \in T u$ and

$$
\pi^{*}(X, 0,0,0)=X \text {. }
$$

Thus $T p \subset \pi^{*} T u$ and therefore

$$
T p=\pi^{*} T u=\pi^{*} \Gamma_{u}^{h}
$$

$\pi^{*}$ will be isomorphism if we show that it is one to one. Let $X, Y \in \Gamma_{u}^{h}$ all arbitrary and

$$
\pi * X=\pi * Y \Longrightarrow \pi *(X-Y)=0 \Longrightarrow X-Y \in L_{u}^{v} \text {. }
$$

Since $X, Y \in \Gamma_{u}^{h} \Rightarrow X-Y \in \Gamma_{u}^{h}$ and consequently

$$
X-Y=0 \Longrightarrow X=Y \text {. }
$$

Hence $\pi^{*}$ is the isomorphism and the theorem is proved.

\section{REFERENCES}

[1] Bishop, R. L., And Critterdon, R. J. Geometry of manifolds, vol. XV. Academic Press, New York-London, 1964.

[2] Воотнвy, M. M., And Wong, R. C. On contact manifolds. Ann. of Math 68 (1958), 391-404.

[3] Das, L., AND Nivas, R. On certain structures defined on the tangent bundle. Rocky Mountain Journal of Mathematics 36 (2006), 885-892.

[4] KAsPer, U. Fibre bundles: An introduction to concepts of modern differential geometry. In Geometry and Theoretical Physics, J. Debrus and A. C. Hirshfeld, Eds. Springer, Berlin, Heidelberg, 1991.

[5] KHAN, M. N. I., AND Jun, J. B. Lorentzian almost r-para-contact structure in tangent bundle. fournal of the Chungcheong Mathematical Society 27 (2014), 29-34.

[6] Kobayashi, S., ANd Nomizu, K. Foundations of Differential Geometry. Inter Science Publishers, John Wiley and Sons, New York, 1963.

[7] Koyabashi, S. Principal bundles with the 1-dimensional toroidal group. Tohoku Math. F. 8 (1956), 29-45.

[8] Mатsumoто, K. On lorentzian paracontact manifolds. Bull. of Yamagata Univ. Nat. Sci. 12 (1989), 151-156.

[9] TALEB, K. Almost product structure in principal fibre bundle over almost para contact manifolds. f. Univ. Kuwait Science 16 (1989), 215-220.

[10] Warner, F. W. Foundations of Differentiable Manifolds and Lie Groups. Springer-Verlag, New York, 1971.

Open Access statement. This is an open-access article distributed under the terms of the Creative Commons AttributionNonCommercial 4.0 International License (https://creativecommons.org/licenses/by-nc/4.0/), which permits unrestricted use, distribution, and reproduction in any medium for non-commercial purposes, provided the original author and source are credited, a link to the CC License is provided, and changes - if any - are indicated. 